\title{
Granzyme B expression in T-regulatory cells is a strong predictor of acute graft- versus-host disease after day +30 in patients with classic immunosuppression after allo-HSCT
}

Mikhail Y. Drokov, Elena N. Parovichnikova, Julia O. Davydova, Larisa A. Kuzmina, Irina V. Galtseva, Nikolay M. Kapranov, Vera A. Vasilyeva, Darya S. Dubnyak, Olga M. Koroleva, Ekaterina D. Mikhalcova, Natalia N. Popova, Valery G. Savchenko National Research Center for Hematology, Ministry of Health, Moscow, Russian Federation

Contact: Dr. Mikhail Drokov: Moscow

E-mail: mdrokov@gmail.com

\section{Introduction}

Granzyme B is a serine protease commonly found in cytoplasmic granules of cytotoxic lymphocytes and natural killer cells. It is secreted with the pore-forming protein perforin and mediates apoptosis in target cells. Granzyme B mediated cytolysis is one of the regulatory mechanisms, together with IL-2 receptors (CD25) by which T-regulatory cells (T-regs) influence T-effectors. Despite the well-known fact that T-regs participate in pathogenesis of aGVHD, and their levels after allo-HSCT inversely correlate with a probability of aGVHD, the data about functional status of T-regs and aGVHD is still limited.

\section{Patients and methods}

Peripheral blood samples were collected in EDTA-tubes at day +30 after allo-HSCT. We use PBMC from 29 patients with hematological malignancies obtained by density gradient media. This method was used due to lymphopenia in this group of patients. Group with no aGVHD consisted of 22 patients $(\mathrm{AML}=12, \mathrm{ALL} \mathrm{n}=5, \mathrm{LPD}=1, \mathrm{CML}=2, \mathrm{AA}=1, \mathrm{MDS}=1)$ after allo-HSCT (17, MUD; 5, mismatched unrelated donor). MAC conditioning was used in 17 cases, whereas 5 patients received RIC. The group of aGVHD after day +30 included 7 patients. (AML $=4$, ALL $n=3$ ) after allo-HSCT from MUD $(n=5)$, mismatch unrelated donor $(n=1)$ and 1 recipient of HLA-identical sibling donor. Two patients received a myeloablative conditioning (MAC), and 5 patients received reduced-intensity conditioning regimens (RIC). All the patients in both groups received standard immunosuppression $(\mathrm{MMF}+\mathrm{CSA}+\mathrm{ATG})$. All the patients developed II-IV grade
aGVHD (grade II, 1; grade III, 4; grade IV, 2 patients), with a median onset on day +50 (34-150). Anti-CD4-APC-Cy7, anti-CD25-APC, anti-CD127-FITC and anti-Granzyme B-PE (Becton Dickinson, USA) antibodies were used to determine T-regulatory cells population. A Bland-Altman plot (difference plot) was used to test the agreement between the two different T-reg assays (CD4+CD25high, CD4+CD25highCD127low), and no significant differences were found $(\mathrm{p}=0.942)$. Due to this fact, CD4+CD25high cells were identified in our experiments as T-reg cells (Gregg et al., 2005). 30,000 CD4+ cells were analyzed on a BD FACSCanto II to achieve sufficient statistical power (Becton Dickinson, USA).

\section{Results}

As seen from Fig. 1, the Granzyme B levels were higher in patients who never developed aGVHD. Fig. 2 shows that a mean percentage of Granzyme B positive T-regs in GVHD-free patients was $7.26 \pm 1.89 \%$, thus being significantly higher than in group who developed aGVHD after day $+30(2.04 \pm 0.93 \%$; $\mathrm{p}=0.02$ ). Noteworthy, according to our previous data, bacterial or viral infections (e.g CMV) and HLA-mismatches does not affect the Granzyme B expression levels in T-regs. Using ROC curve analysis (Fig.3), we obtained the area under curve (AUC) value of 0.74 .

\section{Conclusion}

Our data show that Granzyme B in T-reg cells after allo-HSCT in patients at a standard immunosuppressive therapy may predict aGVHD onset after day +30 , with sat- 
isfactory analytic results (AUC=0.74; cutoff value, $4.15 \%$; sensitivity, $85.71 \%$; specificity, $45.45 \%$ ). According to data of our transplant center (2006-2016), the Granzyme B-based strategy may be helpful for prediction of up to $47.3 \%$ of aGVHD cases. Disclosures: No relevant conflicts of interest to declare.

\section{Keywords}

Allo-HSCT, Granzyme B, T regulatory cells, acute graft-versus-host disease, predictive marker.
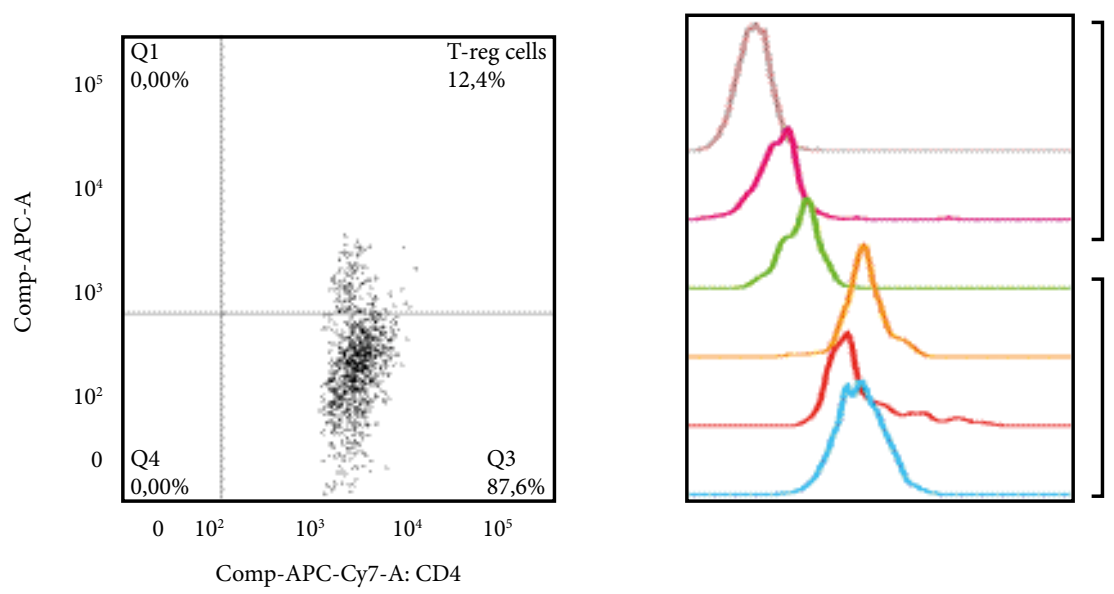

Patients with aGVHD after day +30

Patients without aGVHD in future

Figure 1: Typical patterns of Granzyme B expression (right; shown on horizontal axis), and Treg subset (left; upper right quadrant) on day +30 after allo-HSCT with a standard immunosuppression regimen.

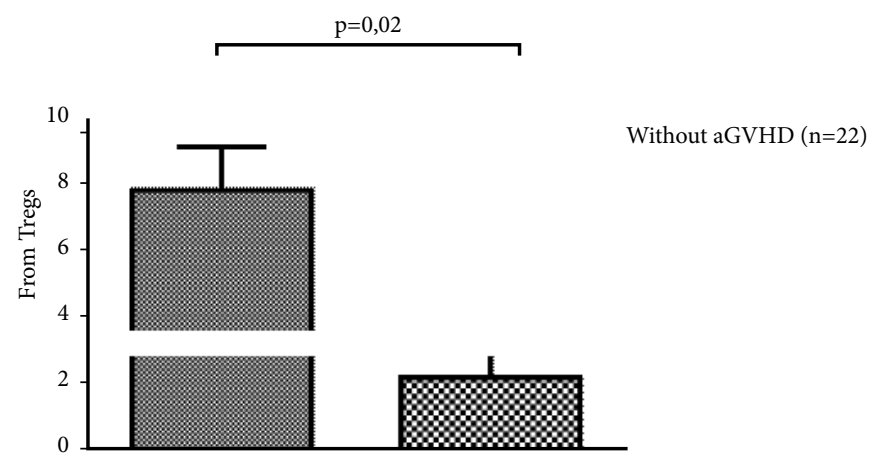

Figure 2: Percentage of Granzyme B positive T-reg cells in aGVHD-free patients and patients who developed aGVHD after day +30 .

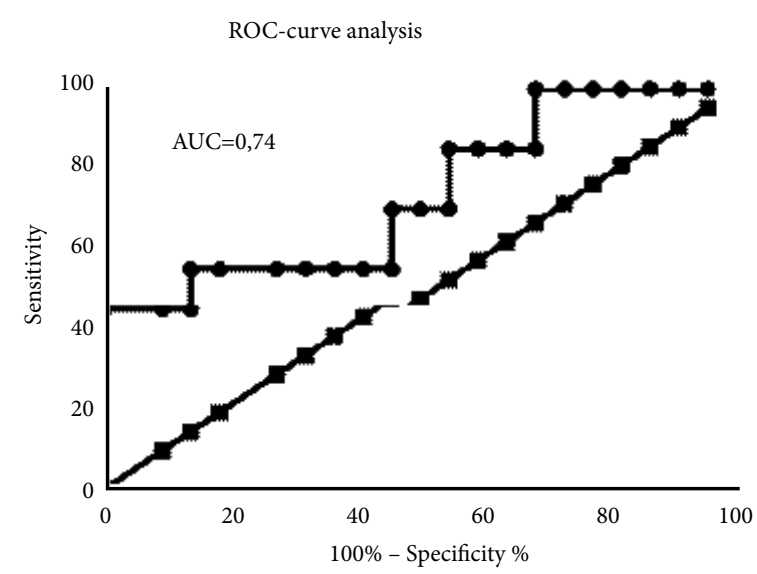

Figure 3: ROC-curve analysis of Granzyme B expression in T-regs as aGVHD predictor. 


\title{
Использование экспрессии гранзима В в Т регуляторных клетках у пациентов с «классической» иммуносупрессией на +30 день после алло-ТГСК в качестве предиктора развития острой реакции «трансплантат против хозяина»
}

\author{
Михаил Ю. Дроков, Елена Н. Паровичникова, Юлия О. Давыдова, Лариса А. Кузьмина, Ирина В. Гальцева, Ни- \\ колай М. Капранов, Вера А. Васильева, Дарья С. Дубняк, Ольга М. Королева, Екатерина Д. Михальцова, Ната- \\ лья Н. Попова, Валерий Г. Савченко
}

Национальный гематологический научный центр, Москва, Россия

\section{Введение}

Гранзим В представляет собой сериновую протеазу широко встречающуюся в гранулах цитотоксических $\mathrm{T}$ лимфоцитов и натуральных киллеров. Он секретируется совместно с формирующим поры белком перфорином, что приводит к индукции апоптоза в клетках-мишенях. Цитолиз клеток опосредуемый гранзимом В, является одним из механизмов, который используют Т регуляторные клетки (Трег) для воздействия на цитотоксические лимфоциты. Несмотря на хорошо известный факт, что Трег участвуют в патогенезе острой реакции трансплантат против хозяина (оРТПХ) и их количество после алло-ТГСК обратно коррелирует с вероятностью развития оРТПХ, данные о функциональном состоянии Трег при оРТПХ по-прежнему ограничены.

\section{Пациенты и методы}

Образцы периферической крови от 29 больных с гемобластозами отбирали в пробирки с ЭДТА на +30 день после алло-ТГСК. В связи с выраженной лимфопенией, что затрудняло анализ субпопуляции Трег, у этой группы пациентов для исследования выделяли достаточное количество мононуклеаров на градиенте плотности ( $\rho=1,077$ г/ мл). В первую группу - без каких-либо проявлений оРТПХ за время наблюдения было включено 22 пациента (ОМЛ $\mathrm{n}=12$, ОЛЛ $\mathrm{n}=5$, ЛП $\mathrm{n}=1$, ХМЛ $\mathrm{n}=2, \mathrm{AА} \mathrm{n}=1$, МДС $\mathrm{n}=$ 1). Из них 17 алло-ТГСК были выполнены от неродственных совместимых доноров, в 5 случаях - от неродственных несовместимых доноров. В 17 случаях был использован миелоаблативный режим, 5 пациентам был проведен режим кондиционирования пониженной интенсивности. Во вторую группу пациентов, с развитием оРТПХ после дня +30 , были включены 7 пациентов: с ОМЛ $(\mathrm{n}=4)$ и ОЛЛ $(\mathrm{n}=3)$. Из них в 5 случаях алло-ТГСК проводилась от неродственного совместимого донора, в одном - от неродственного несовместимого донора, и у 1 пациента донором был HLA-идентичный сиблинг. Двум пациентам был проведено миелоаблативное кондиционирование, а
5 пациентам - режим с пониженной интенсивностью. Все пациенты в обеих группах получали стандартную профилактику РТПХ (ММФ + ЦСА + АТГ). У всех пациентов во второй группе (с оРТПХ после +30 дня) была констатирована оРТПХ II-IV степени (II ( $\mathrm{n}=1)$; III $(\mathrm{n}=4)$; IV $(\mathrm{n}=2)$ ). Медиана развития РТПХ составила 50 сут. (34-150). Для определения доли гранзим В-позитивных Трег были использованы антитела к CD4-APC-Cy7, CD25-APC, CD127FITC и Granzyme B-PE (Becton Dickinson, USA). Учитывая наличие нескольких методов для идентификации Трег: а именно как CD4+CD25high и CD4+CD25highCD127low популяции, с целью сравнения этих двух способов измерения нами предварительно был выполнен анализ по методу Блэнда-Алтмана. Сравнительный анализ этих результов не показал различий по этим двум методам при измерении доли гранзим В-позитивных Трег $(\mathrm{p}=0,942)$. В связи с этим, в дальнейших экспериментах, с целью повышения воспроизводимости результатов, Трег идентифицировались как клетки с фенотипом CD4+CD25high (Gregg et al., 2005). Для достижения достаточной статистической мощности анализировали $30000 \mathrm{CD} 4+$ клеток. Анализ проводили на проточном цитометре BD FACSCanto II (Becton Dickinson, США).

\section{Результаты}

Из рис. 1 видно, что доля гранзим В-позитивных Трег была выше у пациентов, у которых не наблюдалась оРТПХ. На рис. 2 видно, что доля гранзим В-положительных Трег в группе пациентов, у которых не было отмечено признаков оРТПХ, составила 7,26 $\pm 1,89 \%$, будучи достоверно выше, чем в группе с оРТПХ (2,04 $\pm 0,93 \%$; $\mathrm{p}=0,02)$. Следует отметить, что, согласно нашему предыдущему анализу, бактериальная и/или вирусная инфекция, а также различие по HLA не оказывают влияние на долю гранзим В-позитивных Трег после алло-ТГСК. Далее нами была выполнена попытка использования доли гранзима В в Трег как прогностического маркера оРТПХ после +30 дня. На рис. 3 отображены результаты ROC-анализа. По нашим данным, показатель AUC («площадь под кривой») составил 0,74. 


\section{Выводы}

Согласно полученным нами данным, доля Гранзим B-позитивных Трег у пациентов со стандартной профилактикой, может быть использована для прогнозирования оРТПХ после +30 дня с удовлетворительными результатами (AUC: 0,74; «cut-off»: 4,15\%; чувствительность: 85,71\%; специфичность: 45,45\%). По данным нашего трансплантационного центра за 2006-2016 год, анализ экспрессии гранзима В в Трег как прогностического маркера позволяет предсказать до 47,3\% случаев оРТПХ.

\section{Ключевые слова}

Алло-ТГСК, Гранзим В, Т-регуляторные клетки, оРТПХ, прогностический маркер. 